INTERNATIONAL JOURNAL OF SCIENTIFIC RESEARCH

\title{
A CONSERVATIVE AND COSTEFFECTIVE APPROACH FOR ENHANCING AESTHETICS - A CASE REPORT
}

\section{Dental Science}

Dr. Najaf*

Department of Prosthodontics, Crown \& Bridge and Implantology, AME'S Dental College \& Hospital, Raichur, Karnataka, India. *Corresponding Author

Dr. Sunil V Dhaded

Department of Prosthodontics, Crown \& Bridge and Implantology, AME'S Dental College \& Hospital, Raichur, Karnataka, India.

\section{ABSTRACT}

Management of cases with spacing and fluorosis can be quite challenging but, with the plethora of options available in cosmetic dentistry, it has become relatively easier to manage such cases. Since most of these treatments are demanding and expensive, composite veneers were planned for the patient. Hence, with the objective consideration of possible treatment alternatives to meet patient's aesthetic and economic demands, excellence in dental practice can be delivered.

\section{KEYWORDS}

aesthetics, direct composites veneers, spacing, fluorosis.

\section{INTRODUCTION}

Patients seeking to improve the appearance of their smiles have driven the profession towards more challenges in aesthetic dentistry to answer their needs. One of the main goals of dental treatment is to mimic teeth and design smiles in a most natural and aesthetic manner, based on the individual and specific needs of the patient ${ }^{1}$. More patients are seeking cosmetic dental treatment than ever before. While many options are available to suit various needs and expectations, patient often have concerns regarding clinical procedures, finances and removal of healthy tooth structure to enhance their smiles.

Diastema is presence of a space between anterior teeth. It is a common feature of adult dentition. These spaces usually distort a pleasing smile by concentrating the observer's attention not on the overall dental composition, but on the diastema. ${ }^{3}$ Whereas, dental fluorosis presents with stained and/or spotted enamel. Fluorosis is a dental enamel defect that is caused by excessive systemic use of fluoride. It can be a significant esthetic concern because of the yellow, orange, and/or white discoloration that accompanies it. Superficial fluorosis stains can be removed with a technique called micro-abrasion, which is the selective removal of the stained enamel ${ }^{4}$. There are many methods of treatment for such cases like porcelain laminate veneers, direct bonding and crowns, both with and without orthodontics.

Today with the trend towards minimum intervention in dentistry, composite veneers have become an answer to discoloured anterior teeth and spaces between them. Direct placement of composite veneers has greater control, requires minimal removal of tooth structure and can be modified at any stage. These veneers are easily reshaped and polished, especially in the emergence angle of the crown. Treatment is usually completed in one appointment. In addition, cracks or fractures on the restoration may be repaired intraorally. ${ }^{6}$ This case report illustrates direct composite veneering for restoring aesthetics.

\section{CLINICAL REPORT:}

A 32 year old man reported with the complaint of spaces and discolouration with his upper teeth. Upon clinical examination it was seen that patient had midline diastema with lateral spacing and mild fluorosis in relation to his upper teeth. (Fig 1)

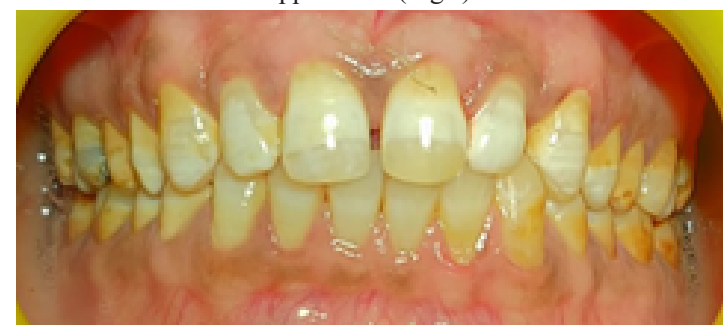

(Fig 1 )

An OPG was then obtained to check for any periapical pathologies, caries and to assess the health of periodontium. Considering the patient's demands like low cost, less time of treatment, noninvasiveness, good outcome and by keeping all treatment options in mind, decision was made. The treatment plan formulated was direct composite veneers. Primary impressions were made using alginate impression material (Dentsply Zelgan Plus Alginate) and diagnostic casts were poured using dental stone. Minimal labial preparation of upper teeth from right premolar to left premolar to be veneered was planned. Additionally, enameloplasty with upper left canine was planned as it was longer than the right canine cervico-incisally, which added to the patients unpleasing smile. Areas of enameloplasty were marked on the diagnostic cast and tooth preparation was carried out on the marked areas of the cast in a controlled manner. A wax-up was fabricated using type II inlay wax to allow the patient to visualize the desired modifications (Fig. 2).

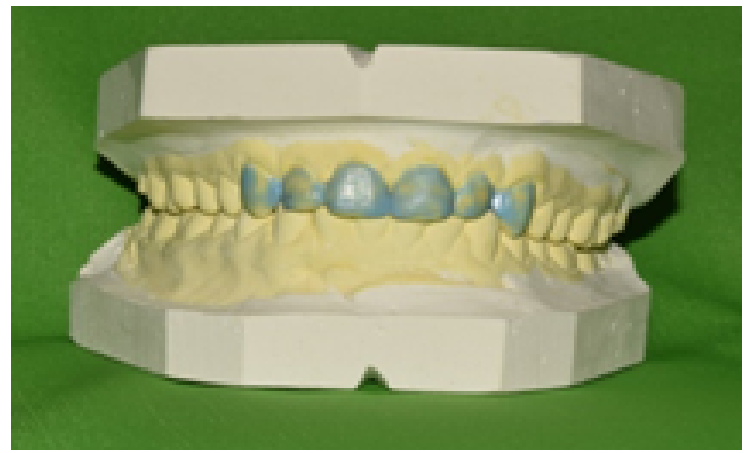

(Fig 2 )

Putty index of the wax mock-up was prepared using Aquasil (Medium body, Dentsply, America). The mock-up was shown to the patient.

In the patient's next visit, minimal preparation involving only enamel on the labial surface of teeth was done. GC Solare universal bond was applied on the prepared labial surface of anterior teeth and cured for 20 seconds. Putty index was then transferred into the patients mouth (Fig 3) and gross build up was done using GC Solare Sculpt- Universally sculptable dental composite.

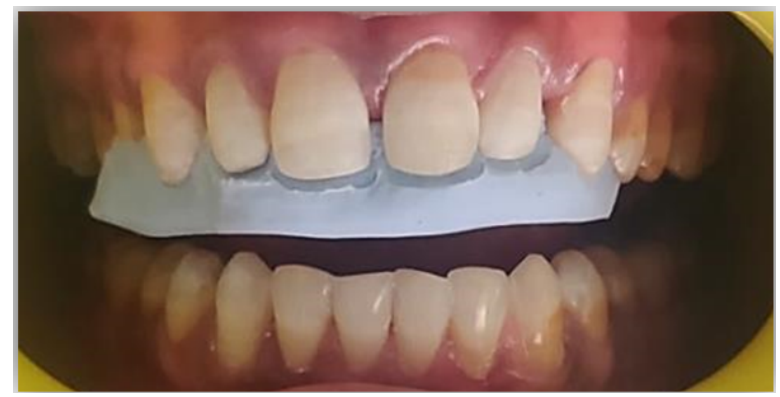

(Fig 3 )

Finally, finishing and polishing of the restoration was done using Shofu rainbow polishing kit with contra-angled low speed micromotor hand piece (Fig 4). 


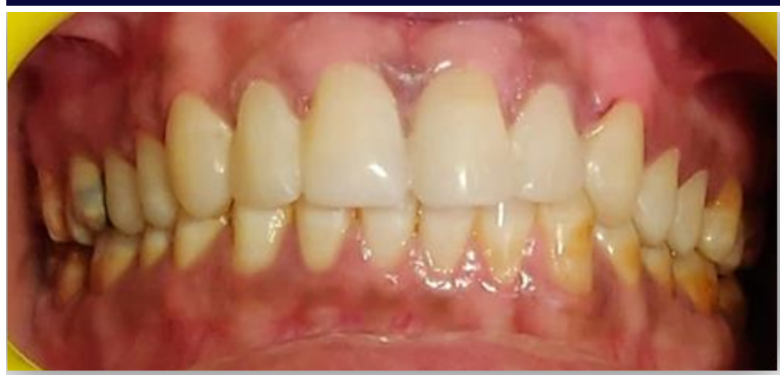

(Fig 4)

Pre-operative and post-operative smiles were evaluated (Fig. 5). Patient was happy with the overall result.
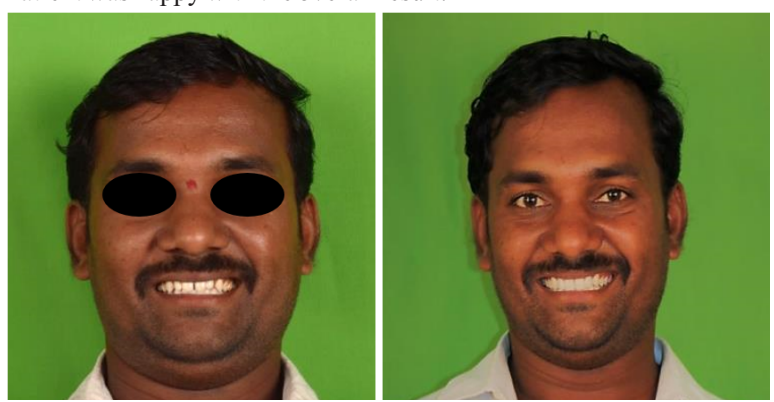

(Fig 5)

\section{DISCUSSION:}

Direct composite veneers can be a choice for teeth with facial surfaces that are malformed, teeth that are discoloured by trauma or stains. They are also indicated incase of eroded and abraded teeth, hypocalcification, diastemas and peg laterals. ${ }^{7}$ Other treatment options like ceramic laminate veneer restorations can also be done as they have advantages like color stability and high resistance against abrasion but they also have some disadvantages such as high cost and long chair time. ${ }^{8}$ Chair side composite restorations have emerged to provide the operator with greater control over the restorative process by sculpting the final restoration to the desired morphology and colour. Due to remarkable improvements in composite resin formulation a new technical and artistic level has been reached. Moreover, it requires minimal removal of tooth structure and can be easily repaired and modified at any stage. In this case direct composite veneers were selected as treatment option, keeping in mind the need and requirements of the patient. Although, indirect veneers could be an option here, direct veneers were preferred because of their less invasiveness, cost effectiveness, less time consumption and patient appointments.

\section{CONCLUSION:}

With the advantages like single appointment for the treatment, cost effectiveness when compared with other options and no need for long laboratory procedures, direct composite veneers remain one of the best treatment choices. Moreover, it's a conservative approach that can satisfy the patient's aesthetic demands while meeting his economic criteria.

\section{REFERENCES:}

Evolution of Aesthetic Dentistry M.B. Blatz1, G. Chiche2, O. Bahat3 , R. Roblee4, C. Coachman 1,5, and H.O. Heymann

2. DiMatteo A. The smile makeover craze: what it means to you and your patients. Inside Dentistry.2006;2(4):45-54

3. Gurel G. Porcelain laminate veneers for diastema closure. In: The science and art of PLV. Ergolding (Germany): Quintessence Publishing; 2003. p. 369-92.

4. Dental Fluorosis André V. Ritter, DDS, MS

5. Beasley WK, Maskeroni AJ, Moon MG, et al. The orthodontic and restorative treatment of a large diastema: a case report. Gen Dent 2004;52(1):37-41.

6. Anterior Space Management: Interdisciplinary Conceptsjerd 5151.15 IYARINT Anterior Space Management: Interdisciplinary Conceptsjerd_5IS ITSAT, DDS, PhD*, CHALERMPOL LEEVAILOJ, DDS, MSD, ABOD, FRCDT $\dagger$

7. Principles of operative dentistry: Sturdevent 5 th Edition.

8. Hickel R, Heidemann D, Staehle HJ, Minnig P, Wilson NHF. Direct composite restorations extended use in anterior and posterior situations. Clin Oral Invest 2004; $8: 43-4$. 